2015

\title{
Parts of the Whole: Quantitative Literacy on a Desert Island
}

Dorothy Wallace

Dartmouth College, dorothy.wallace@dartmouth.edu

Follow this and additional works at: https://digitalcommons.usf.edu/numeracy

Part of the Educational Leadership Commons, and the Higher Education Commons

\section{Recommended Citation}

Wallace, Dorothy. "Parts of the Whole: Quantitative Literacy on a Desert Island." Numeracy 8, Iss. 2 (2015): Article 15. DOI: http://dx.doi.org/10.5038/1936-4660.8.2.15 


\title{
Parts of the Whole: Quantitative Literacy on a Desert Island
}

\begin{abstract}
Some of the specific institutional problems faced by quantitative reasoning courses, programs and requirements arise from the fragile intellectual position of "quantitative reasoning" as an idea, or meme. The process of isolation and reintroduction explains both the proliferation of living species and the way in which some difficult ideas take their place in a culture. Using evolutionary explanations as metaphor and the Copernican revolution as an example of a difficult idea, we draw lessons that can be applied to the "quantitative reasoning" meme, including the function of the National Numeracy Network as an island of protected discourse favoring the growth of the QR meme. We conclude that the mission of the National Numeracy Network should focus on attributes of that island, and in particular extend the mission beyond being a network, to being an actual community.
\end{abstract}

\section{Keywords}

quantitative literacy, quantitative reasoning, numeracy, memetics

Creative Commons License

(c) (1) (9)

This work is licensed under a Creative Commons Attribution-Noncommercial 4.0 License

\section{Cover Page Footnote}

Dorothy Wallace is a professor of mathematics at Dartmouth. She was 2000 New Hampshire CASE Professor of the Year, and the lead PI of the seminal NSF project, Mathematics Across the Curriculum. She recently finished a text in mathematical biology for first-year students, "Situated Complexity." She was a charter board member of the National Numeracy Network and is now co-editor of this journal. 


\section{Parts Of The Whole A Column by $\mathrm{D}$. Wallace}

The problem of how best to improve the numeracy of a society is a thorny one, embracing the learning process of a single student but rising in scale to include the management and alteration of an entire system of education. With the issue of quantitative literacy always in mind, this column considers various aspects of the systemic workings of education, the forces acting on classrooms, teachers and students, and mechanisms of both stasis and change.

\section{Quantitative Literacy on a Desert Island}

A university wishes to create a quantitative reasoning requirement for students, but even a faculty committee of only ten people can't agree on exactly what it is. Another university has quantitative reasoning courses, all of which are in the mathematics department, causing both students and faculty to regard them (quite reasonably) as just another kind of mathematics course. A third university also has quantitative reasoning courses, but these are offered through a center that functions independently of any academic department, making these courses into institutional orphans staffed by faculty who get less recognition (and probably pay) than their departmental equivalents. A fourth university has courses emphasizing quantitative reasoning in a variety of academic departments, competing for resources with courses directly serving a variety of majors, and offered out of the largesse of each departmental budget.

These scenarios illustrate two points. First, there are a lot of ways QR could fit into an institutional setting. Second, the current position of QR at many institutions is fragile. It suffers from the fact that, as a recently introduced meme, it is still in a culturally weak position. This weakness is, of course, normal for a new idea. But it is one of the core problems behind each of the scenarios above. The desire to help this particular meme to grow in strength is one of the reasons for the existence of the National Numeracy Network, even if it is not usually stated that way. So it is worth asking how one might go about helping a meme to grow.

Once the earth stood immobile at the center of the universe. It no longer does so, requiring of us a change in worldview of literally mythic proportion. Perhaps the Copernican solar system is the prime example of how a radically new idea moves into a culture. Many have studied this particular example, but few have dared to draw any generalizations from it. For better or worse, it is the business of this column to draw generalizations. Because we are using ecology as our metaphor we will once again visit that lovely subject and begin with its 
generalizations, returning later to our cosmological example, and in the end to the problem of strengthening "quantitative reasoning" as a meme.

\section{Isolation and Re-introduction of Species}

How then, do new species arise? Mathematics has less to offer us here, but ecology offers a lot. When a population of finch is divided and sent to live on two different islands, an amazing thing happens. Random mutation and slight differences in habitat will cause the two new populations of finch to differentiate from each other, both in external appearance and in feeding habits. This is the brilliant observation of Darwin that is yet to be contradicted. Sometimes the two populations come together again, perhaps by migration or by the actual joining of islands via a new geologic formation such as a land bridge. Simple mathematical models would say that one of the two species would probably die out when put in direct competition with the other. Yet nature has found another path. Because the two populations now have different feeding habits and refuse to interbreed, they can coexist. They have, by modifying themselves, taken one niche and split it in half.

The ecologists call this process "isolation and reintroduction," and it accounts for the many grass eaters on the prairie. ${ }^{1}$ Some are eating grass, some leafy greens, others woody shrubs. Some browse indiscriminately. Some pass through the land at different times of year. Their habits allow them to coexist and their physiological differences prevent them from interbreeding. Ecologists believe that most of this diversity arose through repeated processes of isolation and reintroduction as climate change and other external phenomena repeatedly separated and brought together populations. Each new species has its own specialized lifestyle. Its relationships to the other species are unique to itself.

The process of isolation and reintroduction is simple enough, but to apply it to the world of ideas we must have an idea of what constitutes an idea's "habitat". Here we enter the realm of cognitive science, where many models have been proffered. The most useful of these, both from the practical pedagogical viewpoint and from the ecological one we are exploring here, is the notion of a "schema." Let us imagine how a child learns the concept of a "circle". First, there must be many circular objects in the child's experience. "Circle" is first of all a category that contains these objects. Later the child will learn the definition of a circle as points equidistant from a given one. Now this definition is

\footnotetext{
${ }^{1}$ Colinvaux, Paul A. Why big fierce animals are rare: an ecologist's perspective. Princeton University Press, 1979.

2 Duckworth, Eleanor. The having of wonderful ideas and other essays on teaching and learning. Teachers College Press, 1996.
} 
connected to the concept of circle. Much later the child may learn the algebraic equations describing a circle and those equations will "be" circles ever after. Pedagogically, we measure the depth of the student's understanding of the concept of circle by how rich its set of connections is-- how the student recognizes the concept in a variety of contexts. The success of the idea of "circle" is dependent on how many other ideas lead to it through the neural network in the student's head. We could say that this set of connections constitutes the "habitat" for the idea. Like the situation for a species, no two ideas have identical schema, else they would be the same idea. Writ small in the context of individual cognition, the habitat for an idea is called a schema.

On a larger scale, scholars also describe the connections between ideas within an entire culture. Gerald Holton, a historian of science, noted certain key ideas that seemed to be connected to huge quantities of other ideas throughout a culture. These he termed "thema."3 Earlier, Thomas Kuhn called them "paradigms" and asserted that the history of scientific thought is punctuated with "paradigm shifts," of which the Copernican revolution was one. ${ }^{4}$

When, on the scale of a population, the connections between common concepts are revised on a large scale, we see a paradigm shift, or a change of thema. This is indeed suggestive of what happens when a new species is successfully introduced into an ecosystem. The relationships must all change slightly to accommodate the new species in such a way that it is not in direct competition for someone else's niche, else one of the two will probably become extinct. If the original species occupying that niche is lost, then many more may go down as well. So, a previous niche must be somehow partitioned to make space for the newcomer, much as a large and growing family keeps fitting more beds into limited space. So we see some comparable ecological and memetic phenomena on a macroscopic scale. Therefore let us look at the mechanism of isolation and reintroduction to see if it is at work in the example of the Copernican revolution.

\section{Copernicus}

The concept of a heliocentric universe was in play before Copernicus, and indeed one of Copernicus' first statements in De revolutionibus orbium coelestium attributes this belief to the ancients, thus throwing out a very valuable edge connecting his work to highly valued Greek wisdom. ${ }^{5}$ Yet his book was not

\footnotetext{
${ }^{3}$ Holton, Gerald, and James L. Park. "Thematic origins of scientific thought: Kepler to Einstein." American Journal of Physics 42.4 (1974): 346-347.

${ }^{4}$ Kuhn, Thomas S. The structure of scientific revolutions. $50^{\text {th }}$ anniversary edition. University of Chicago Press, 2012.

${ }^{5}$ Copernicus, Nicolaus. "De revolutionibus orbium coelestium, 1543." On the revolutions (1959).
} 
published for many years, growing to its full glory in protected obscurity. Had the heliocentric universe come into immediate competition with the theologically correct earth centered universe, chances are good that it would have been nipped in the bud. Let us explore the nature of the isolated intellectual island in which these ideas grew.

One strong intellectual division at the time was between the various branches of study, which were arranged in a hierarchy with theology at the top, followed by philosophy. Mathematics and astronomy were several rungs down the ladder. Long after Copernicus' works were published, Galileo would give up a position as mathematician for a position as court philosopher-- a political move that would immediately raise his intellectual status. But Copernicus remained a mathematician, and as such, was able to postulate a heliocentric model in order to make his theorems possible and perhaps to make his calculations easier. Copernicus really believed his model, as is evident from the first book of his complete work, but many were quick to point out that belief was unnecessary in order to appreciate the ease with which his hypothesis "saved appearances" by accurately predicting certain qualitative observations. The introduction by Osiander, added without Copernicus' permission, goes to great lengths to point out that the heliocentric model is only a useful mathematical hypothesis and should not be construed as contradicting the known assertions of philosophy. Osiander is protecting Copernicus' ideas from direct competition with those of philosophy and theology. As long as his ideas lived in the isolated intellectual fields of astronomy and mathematics, they were free to propagate on their island, being circulated and discussed among those scholars all over Europe.

In the Ptolemaic model with the earth at the center of the universe and each planet held in its own crystalline sphere, many possible planetary configurations are a priori possible. There is no physical reason why Venus, for example, must remain in close proximity to the sun at all times. On the other hand, if one postulates the sun at the center, with Venus orbiting it and the Earth even farther away, it is possible to prove a theorem that constrains Venus' apparent position when viewed from the Earth. She can no longer stray more than some fixed arc from the sun. Copernicus proves this theorem in book 6 of his work, explaining through three-dimensional geometry an astronomical fact observed since the dawn of time. Now, that is "saving appearances" in a way that would have very strong appeal to mathematicians of the time, steeped in the books of Euclid and practicing astronomy on the side as they often did. These mathematicians read Copernicus carefully, noting every error and criticizing not only the astronomy but also often the mathematics. Viète, often called the "father of modern algebra", described Copernicus as a poor calculator and even worse geometer, 
disliking his tables of sine and cosine. Kepler published a volume showing the mathematical equivalence of the two systems. So, we see a very healthy interactive niche for Copernicus's ideas in the isolated world of Renaissance mathematics. While it occupied this niche, the heliocentric theory was most definitely not a model of physical reality but merely a useful mathematical hypothesis for predicting planetary locations.

In this part of the story of the heliocentric universe we see the process of isolation at work in exactly the same way as it would for biological species. What was formerly one niche-- a model of the universe that could be used to make mathematical predictions-- is now two niches. The idea of a model of reality has been separated from its mathematical consequences and two models are simultaneously in play. Both could be models of reality, both could be used to make predictions. But they have diverged in form and also diverged in their relationship to other ideas of the culture, as a divided population would be expected to do. They were allowed by the system to diverge in this fashion because they were isolated from one another, so that the culture at large never needed to make a direct choice between the two.

Isolation allows a new idea to grow unfettered. Reintroduction of this idea to the culture at large introduces new constraints, requires readjustment of all the other ideas connected to it, and is generally a much more chaotic process. In the biological world, when a pair of divergent species are reintroduced to each other, they often diverge further from each other to protect their niches. In the case of the Copernican system, the process of reintroduction may have been gradual but certainly had notable benchmarks in the publication of the Galilean dialogue, "Concerning the Two Chief World Systems", heresy. Galileo unambiguously argues the case for the Copernican system as a true model of the universe, and being the idea-monger he was, published his dialogue in vernacular Italian. Now the Copernican system is in immediate conflict with the Catholic Church, and is pitched into a web of related ideas and circumstances that include the Protestant reformation, the Inquisition, Aristotelian physics and the Thirty Years War. The exact pathway by which the heliocentric system continues to grow in importance is murky and the stuff of many histories. As with the reintroduction of species, the particulars are difficult to predict. What matters is the outcome.

The Copernican system cannot triumph completely over the Ptolemaic without rewriting all of religion, but through a continuing process of diversification both ideas might continue to exist as useful approaches for

\footnotetext{
${ }^{6}$ Galilei, Galileo, and Stillman Drake. Dialogue concerning the two chief world systems, Ptolemaic and Copernican. Random House Digital, Inc., 1953.
} 
different circumstances. For the Copernican system to remain a useful scientific approach it must become more than just a mathematical device. It must, in the end, usurp the role of real physical model of the universe. Galileo, desiring this, argues forcefully that biblical references to the motion of the sun are mere rhetorical devices, and that they, and not Copernicus, are in the business of "saving appearances". The daily rotation of the sun becomes mere appearance. God is excused for describing physical reality in terms understandable to laymen. Instead of philosophy, the predecessor to physics, remaining separated from mathematics, Galileo insinuates that theology should instead be more separate from philosophy. Galileo is playing with the connections between ideas in an attempt to rearrange their relationships to make room for the new idea. In Copernicus, we see isolation of an idea. In Galileo, we see the reintroduction of the ideas to the rest of the world picture at the time.

Now that the argument is over we can see how the system rearranged itself. The Ptolemaic model is still used for the purpose of calculating planetary positions for the next several hundred years. It is the model that most easily saves the appearances. Religious assertions also fall into the category of "saving appearances". The Copernican model becomes the physics, at least until it becomes possible to think the difficult thought that perhaps there is no center of the universe. We still happily speak of the sun travelling through the sky, even though it contradicts the way we understand our own universe. Both ideas have their place, their niche, and can coexist indefinitely in our intellectual ecosystem as a result.

This example shows the validity of thinking in terms of ecological metaphors when studying the propagation of ideas. Ideas do diversify to create new ideas. Difficult or unpopular ideas can thrive in isolation until they are strong enough to be reintroduced to the culture at large. All of mathematics is full of examples of research paths followed in relative obscurity until some connection appears with physics, whereupon they become thriving ventures complete with course offerings, Ph.D. theses, dedicated journals and access to grant money. Those obscure research paths function as a meme-bank for the culture at large, as so many islands of diversity ready to offer something useful when the culture is ready for it.

The example of Copernicus also illustrates the importance of ideas growing in strength before being introduced to the culture at large. In that example, strength came from connections to other ideas: astronomical facts, Newtonian physics, Euclidean geometry, and even stylistically to Euclid's Elements. Strength also came from the many conversations about the heliocentric system among a variety of highly educated professionals in many countries. 


\section{Lessons to be Learned}

Now we must turn to the important question of how to strengthen "quantitative reasoning" as an idea deserving cultural respect and attention, so that those implementing QR courses, programs and requirements are in a stronger philosophical position in their institutions. Drawing on the discussion so far, we would want to create a space where QR could be discussed, defined, adjusted and argued about, but where the basic value of QR would go unquestioned. The National Numeracy Network tries to be such an island, with this journal as one of its vehicles for discussion. Second, there should be individuals engaged in this discussion who come from a wide variety of professional backgrounds. It is for precisely this reason that the National Numeracy Network is not under the umbrella of some existing disciplinary organization (such as the Mathematics Association of America, a natural candidate). It is critical for the growth, stability, and strength of "quantitative reasoning" (the meme) that its connections extend, and are perceived to extend, beyond any single discipline. Third, there should be a large number of such individuals. A large group of people held together by a common interest is called (at least in my groovy part of the country) an "intentional community".

At this point in the history of the QR movement, it is the community aspect that seems most lacking. In addition to a common interest, communities need structure, opportunities to gather together, a sense among members that they are cared for by the group, and even some small customs that identify members and mark the passing of days. The National Numeracy Network would do well to set an agenda for creating this environment, thereby extending its mission from creating a network of $\mathrm{QR}$ practitioners, to creating an actual community. It would be wrong to draw strong conclusions from a single example, but it may be that building and strengthening this community is the necessary next step in strengthening the cultural identity of "quantitative reasoning," which in turn will strengthen its various incarnations at the secondary and college level. 